\title{
Single-beam spectrally encoded color imaging
}

\author{
Mitsuhiro IKUtA ${ }^{1}$, Dongkyun KAng ${ }^{2,3}$, Dukho Do², Adel Zeidan², \\ GUILLERMO J. TEARNEY2,4,5,*
}

\author{
${ }^{1}$ Healthcare Optics Research Laboratory, Canon U.S.A., Inc., Cambridge, MA 02139, USA \\ ${ }^{2}$ Wellman Center for Photomedicine, Massachusetts General Hospital, Boston, MA 02114, USA \\ ${ }^{3}$ College of Optical Sciences and Department of Biomedical Engineering, University of Arizona, Tucson, AZ 85721, USA \\ ${ }^{4}$ Department of Pathology, Massachusetts General Hospital, Boston, MA 02114, USA \\ ${ }^{5}$ Harvard-MIT division of Health Science and Technology, Cambridge, MA 02139, USA \\ *Corresponding author: gtearney@partners.org
}

Received XX Month XXXX; revised XX Month, XXXX; accepted XX Month XXXX; posted XX Month XXXX (Doc. ID XXXXX); published XX Month XXXX

We have developed a new method of conducting spectrally-encoded color imaging using a single light beam. In the new method, a single broadband light beam was incident on a diffraction grating, where the overlapped 3 rd order of the red, 4th order of the green, and 5th order of the blue spectral bands were focused on a line illuminating tissue. This configuration enabled each point on the line to be illuminated by three distinctive wavelengths, corresponding to red, green, and blue. A custom grating was designed and fabricated to achieve high diffraction efficiencies for the wavelengths and diffraction orders used for color spectrally-encoded imaging. A bench system was built to test the new spectrally-encoded color imaging method. For a beam diameter of $174 \mu \mathrm{m}$, the bench system achieved 89,000 effective pixels over a 70 circular field. Spectrally-encoded color images of excised swine tissue revealed blood vessels with a similar color appearance to those obtained via a conventional color camera. Results suggest that this single-beam spectrally-encoded color method is feasible and can potentially simplify color spectrally-encoded endoscopy probe designs. (C) 2018 Optical Society of America

OCIS codes: (170.2150) Endoscopic imaging; (170.3880) Medical and biological imaging

http://dx.doi.org/10.1364/OL.99.099999

Miniature endoscopy technologies have the potential to provide safer and less traumatic endoscopic imaging and treatment. Several technologies have been demonstrated to conduct endoscopic imaging through small probes [1-5]. Spectrally encoded endoscopy (SEE) is a miniature endoscopy technology that uses a miniature grating to image a line of the tissue without having to use beam scanning devices or fiber bundles [6]. Previously, a 350- $\mu$ mdiameter SEE device was utilized for in vivo laparoscopic imaging of a mouse [7]. Most SEE devices, however, expend spectral information for spatial encoding and therefore do not provide color images of the tissue. The monochromatic images obtained by SEE may not be sufficient in certain cases when color imaging is required for disease diagnosis. A bench top color SEE method that used three spatially offset light beams was previously demonstrated [8]. However, implementing the three-beam color SEE method as a miniature probe could be challenging, as it mandated use of three separate fibers in the device, making free form probe rotation challenging. Another color SEE method that translated the specimen along the axis of the spectrally-encoded line was demonstrated [9]. The translation-based method could be difficult to utilize during in vivo endoscopic imaging due to the movement and deformation of living tissue in the endoscopic environment. In this paper, we present a new approach for conducting color SEE imaging that uses a single light beam. Since a single light beam is used and the tissue does not need to be translated, the new approach can facilitate development of a miniature color SEE probe that can be used for endoscopic imaging in vivo. In order to demonstrate the feasibility of this single-beam color SEE approach, we built a bench setup and tested imaging resolution and color imaging performance.

The new color SEE method is based on a simple principle that as long as product of the wavelength and diffraction order is kept constant, the diffraction angle remains the same. The diffraction equation is given as

$$
\sin \left(\theta_{i}\right)+\sin \left(\theta_{d}\right)=-m \times g \times \lambda,
$$

where $\theta_{i}$ is incidence angle, $\theta_{d}$ diffraction angle, $m$ diffraction order, $g$ groove density of the grating, and $\lambda$ wavelength. We carefully chose three spectral bands that represent blue, green, and red colors, 408 - $480 \mathrm{~nm}, 510-600 \mathrm{~nm}, 680-800 \mathrm{~nm}$, respectively and three corresponding diffraction orders, $-5,-4,-3$, respectively. As a result, for any wavelength in blue spectral band, there always were wavelengths in green and red spectral bands that shared the same diffraction angle.

In the bench top system used to test this SEE color imaging approach's feasibility (Fig. 1), a supercontinuum laser (EXU-6, NKT; Spectral band $=400-2000 \mathrm{~nm}$; spectral power density $=\sim 1 \mathrm{~mW} / \mathrm{nm}$; 
unpolarized) was used as the light source. Light from the source was filtered by a low-pass filter (cutoff wavelength $=900 \mathrm{~nm}$ ) and coupled to a single mode fiber (630HP, Nufern). Light from the fiber was slightly focused by an aspheric focusing lens (Thorlabs, CFC-2X$\mathrm{A}, \mathrm{f}=2 \mathrm{~mm}$ ) and directed towards a custom diffraction grating (groove density $=800$ lines $/ \mathrm{mm}$ ) positioned $25 \mathrm{~mm}$ away from the lens. The Gaussian beam diameter on the grating was $174 \mu \mathrm{m}$ at 568 $\mathrm{nm}$, the green wavelength used at the center of the field of view. The small beam diameter mimicked the beam diameter that would be required to construct a miniature endoscopic probe. The incident angle on the grating was $70^{\circ}$, which constituted the Littrow condition for the wavelength of $470 \mathrm{~nm}$ at the -5 th order. Three distinctive spectral bands representing blue, green, and red were diffracted at different orders ( -5 th , -4 th , -3 rd $)$ in such a way that the three spectral bands overlapped and illuminated the same line on the tissue. The diffraction angle ranged from 44 to $79^{\circ}$, which made the half field angle $35^{\circ}$.

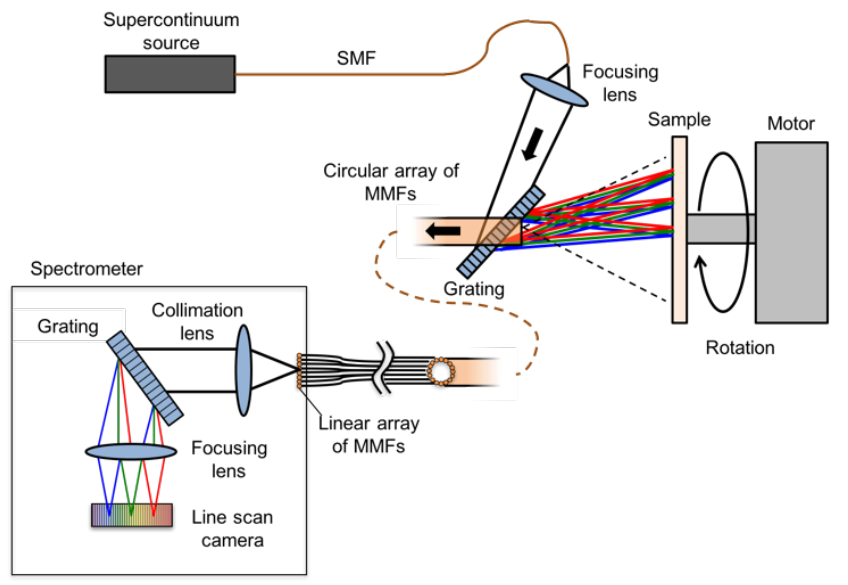

Fig. 1. Schematic of the single-beam color SEE bench top system. SMF single mode fiber; and MMF - multi mode fiber.

Light reflected back from the sample was collected by a circular bundle of multi-mode fibers (FSUA125145185, Polymicro; NA = 0.66 ; fiber core diameter $=125 \mu \mathrm{m}$; number of fibers $=16$ ). The circular fiber bundle was then converted to a linear fiber bundle. Light from the linear fiber bundle was coupled to a custom spectrometer. The custom spectrometer used a collimation lens $(\mathrm{f}=$ $100 \mathrm{~mm}$ ), a diffraction grating (groove density $=1,200$ lines $/ \mathrm{mm}$ ), a camera lens ( $\mathrm{f}=35 \mathrm{~mm} ; \mathrm{f} / 1.4$ ), and a tall-pixel line scan camera (S11490, Hamamatsu; 1024 pixels; pixel size $=24 \times 500 \mu \mathrm{m}$ ). The height of the camera's pixel, $500 \mu \mathrm{m}$, in combination with the spectrometer magnification, 0.35 , allowed effective detection of light from a column of 8 detection fibers. Light detected by the other 8 fibers were not coupled to the spectrometer. The theoretical resolution of the spectrometer was $1.0 \mathrm{~nm}$, which corresponded to spatial resolution of $103 \mu \mathrm{m}$ on the sample plane located at the working distance of $15.5 \mathrm{~mm}$. The sample was mounted on a motor and rotated at $10 \mathrm{rps}$ to acquire two-dimensional, circular SEE images. 1,000 spectrally-encoded lines were acquired per frame. The full field angle was $70^{\circ}$.

A custom diffraction grating was designed to maximize the diffraction efficiencies for the spectral bands and diffraction orders used for single-beam color SEE imaging. A groove density of 800 lines $/ \mathrm{mm}$ (corresponding groove period $=1.25 \mu \mathrm{m}$ ) was used to provide a field angle that was comparable to that of commonly-used video endoscopes, $70^{\circ}$. During the grating optimization process, the following design goals were used: i) high diffraction efficiencies for orders used for color SEE imaging ( -5 th for blue, -4 th for green, and -3rd for red); and ii) low diffraction efficiencies for neighboring orders ( -4 th for blue, -3 rd for green, and -2 nd for red). Fig. 2a shows a schematic of the incident beam and diffracted beams around the grating. The diffraction efficiency of the grating was simulated by the Rigorous Coupled-Wave Analysis (RCWA) method [10]. The refractive index of the grating material was 1.46, similar to the refractive index of a UV-curable epoxy that has been used for miniature grating fabrication [11]. The RCWA simulation results showed that a grating with a groove depth of $1.7 \mu \mathrm{m}$ and a duty cycle of 0.2 (inset of Fig. 2a) produced sufficiently high diffraction efficiency for the color SEE imaging orders and low efficiency for the neighboring orders (Fig. 2b).
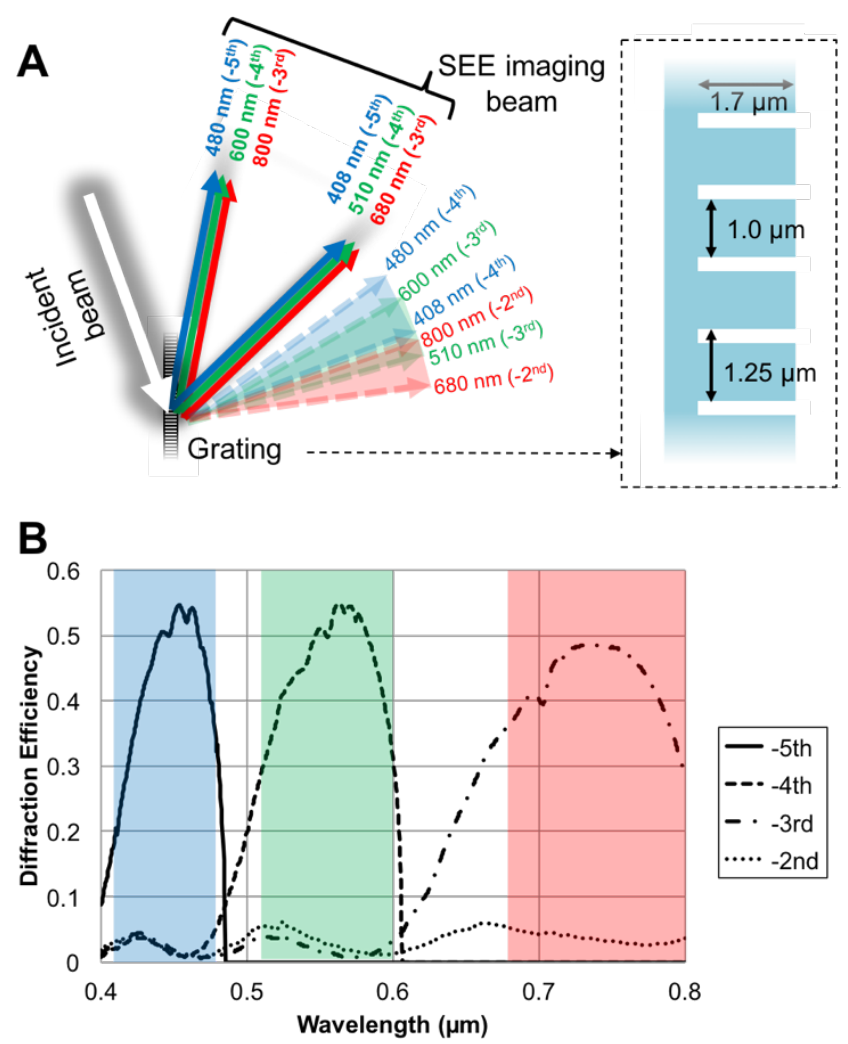

Fig. 2. Schematic of the custom grating's diffraction orders (A) and simulated diffraction efficiencies of the custom grating (B). Inset in (A) schematic of the grating profile.

The grating was made of silica glass by electron-beam lithography and dry etching. The measured values of the diffraction efficiencies were $48 \%, 46 \%$, and $39 \%$ for the $-5^{\text {th }}$ order of $442 \mathrm{~nm},-4^{\text {th }}$ order of $550 \mathrm{~nm}$, and - $3^{\text {rd }}$ order of $740 \mathrm{~nm}$, respectively, which were slightly lower than the simulated values, $51 \%, 51 \%$, and $49 \%$, respectively. Diffraction efficiencies for the neighboring orders were measured to be $12 \%, 14 \%$, and $14 \%$ for $442 \mathrm{~nm}, 550 \mathrm{~nm}$, and $740 \mathrm{~nm}$, respectively, and these measured values were higher than the simulated values, $2.2 \%, 1.7 \%$, and 3.4\%, respectively. Experimental 
deviations from model results were likely due to imperfect grating fabrication.
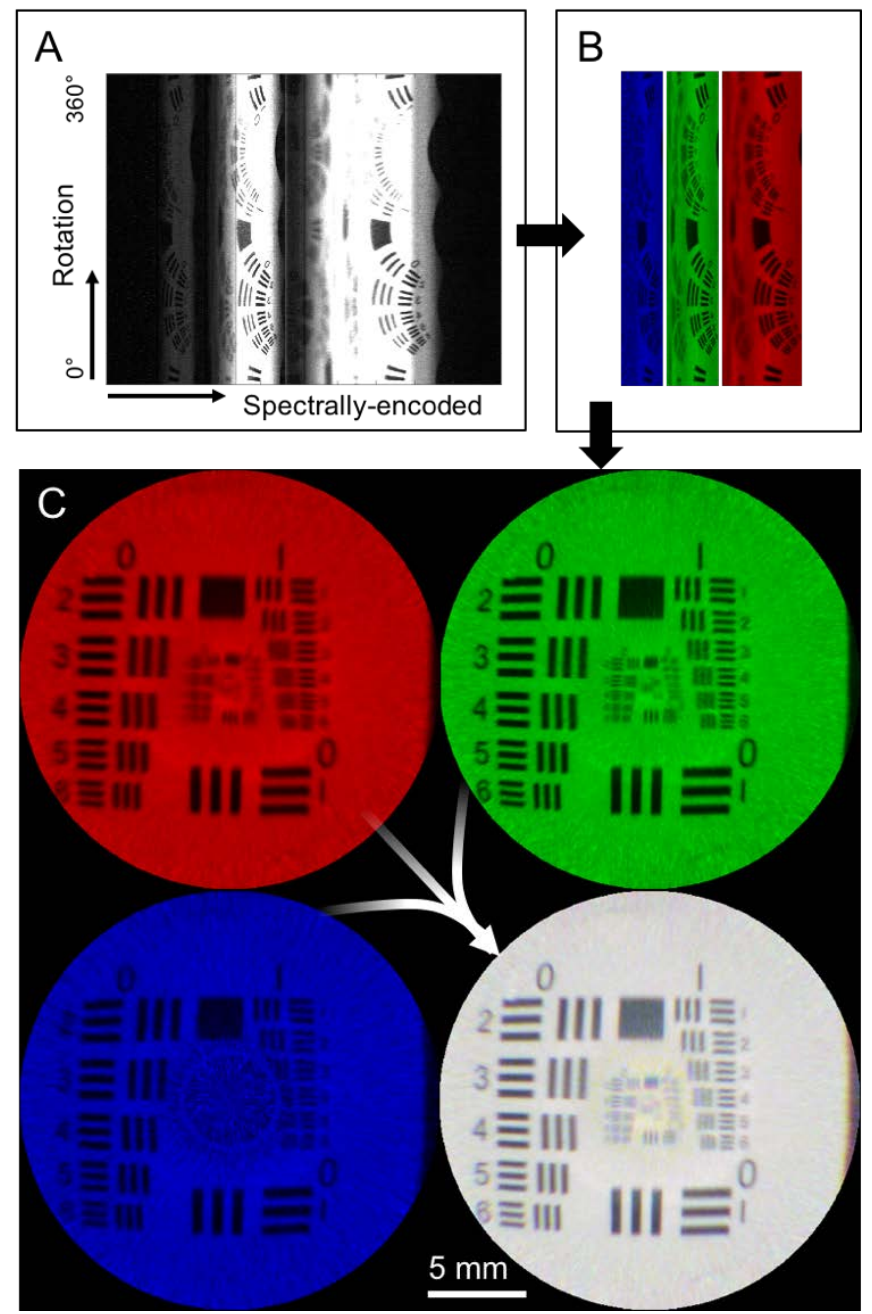

Fig. 3. The process of color SEE imaging processing using data from a 1951 USAF resolution chart. A - monochromatic image obtained from the spectrometer; B - three segmented rectangular images corresponding to each of the red, green, and blue color bands; and C three Cartesian coordinate images combined into the final color SEE image.

Raw spectrometer data (Fig. 3a) was obtained as a twodimensional, monochromatic image with a size of 1024 (spectrometer) $\times 1000$ (rotational) pixels. The horizontal axis in Fig. 3 a represents the spectrally-encoded direction and the vertical axis the rotational direction. The monochromatic image was divided into three segments for the blue, green, and red spectral bands (Fig. 3b). White balancing and spectral compensation were applied on each segment by dividing each acquired spectrum, or each horizontal line, by the spectrum of a reference object [8]. Widths of the three segments, corresponding to light detected from the three color bands, were interpolated to produce equal width images in polar coordinates. The three rectangular images were then converted from polar coordinates into 500x500 pixel, 8-bit Cartesian coordinate images and combined into a 24-bit RGB color image (Fig. 3c).
The number of resolvable points were measured by imaging a USAF resolution target (Fig. 3c). The resolution target was positioned $15.5 \mathrm{~mm}$ away from the grating to mimic a clinicallyrelevant working distance. The lateral resolution was measured by calculating the full width half maximum (FWHM) of the line spread function (LSF) at the center of the spectral band. The number of resolvable points was calculated by dividing the full field angle by the lateral resolution. The spectrometer resolution was measured by coupling light from a mercury-argon calibration source (HG-1, Ocean Optics) into the detection fiber bundle and measuring the FWHM of the peak at the center of the spectrum.

The lateral resolution for the green channel was measured to be $0.31^{\circ}$ for the motor scanning direction and $0.71^{\circ}$ for the spectrallyencoded direction. The measured values were similar to theoretical angular resolutions of $0.25^{\circ}$ and $0.62^{\circ}$. The measured spectrometer resolution was larger than the theoretical value, $2 \mathrm{~nm}$ versus $1 \mathrm{~nm}$, which may have caused the discrepancy between the measured and theoretical lateral resolutions. With the measured angular resolution, the SEE bench system had 226 and 98 resolvable points per full field diameter along the rotational and spectrally-encoded directions, respectively, resulting in 89,000 effective pixels per full circular field. We optimized the focusing lens to position the green beam waist at the resolution target. Due to the chromatic aberration of the focusing lens, the beam waists of the red and blue beams were located away from the target plane. This configuration resulted in better resolution for the green-channel image than those for the red and blue channels. The smallest distinguishable bar in Fig. 3c was 88 $\mu \mathrm{m}$ for green vs. 125 and $140 \mu \mathrm{m}$ for red and blue, respectively. No noticeable cross talk was observed since there was no spectral overwrap in the spectrometer. In addition, the contribution of neighboring orders was minimal due to the low grating diffraction efficiency and low fiber detection efficiency for these neighboring orders. The signal-to-noise ratio (SNR) varied over the field of view due to the non-uniform source spectral density, grating diffraction efficiency, and fiber detection efficiency. The measured SNR values were $13.3 \pm 9.3,19.2 \pm 3.9$, and $18.9 \pm 5.5$ for blue, green, and red channels, respectively.

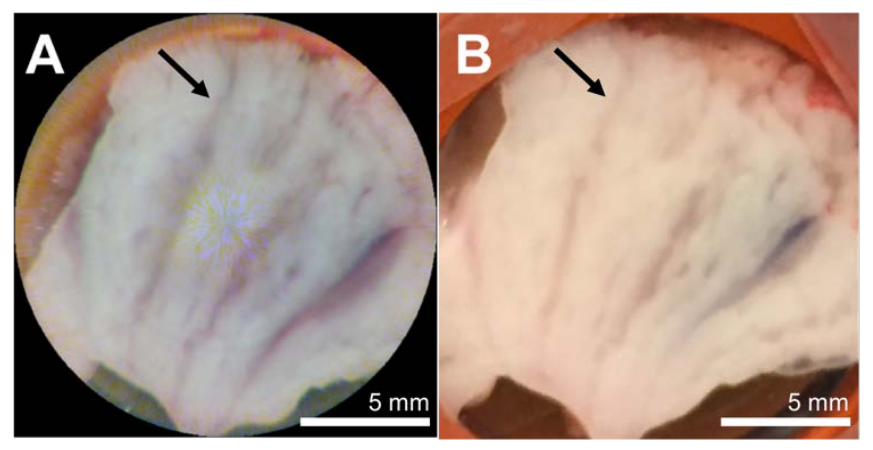

Fig. 4. Color SEE image (A) and conventional color digital photograph (B) of excised swine mesentery. Arrows denote mesenteric vessels.

Tissue imaging performance was tested by imaging excised swine mesentery. The swine tissue was placed inside a tissue container (Coverwell imaging chamber, Grace Bio-labs) and gently pressed under a coverslip. The coverslip was attached to the tissue container using an adhesive tape. The tissue was then mounted on a motor and imaged by the color SEE bench system. The power incident on the 
tissue was $20 \mathrm{~mW}$. The same tissue was then photographed by a smartphone camera (iPhone 6) for comparison. A color SEE image and photo of the swine tissue are shown in Fig. 4. The color SEE image (Fig. 4a) clearly visualized red blood vessels (arrow) against the surrounding tissue. The color appearance of the tissue in the SEE image was similar to that observed in the color camera's image (Fig. 4b).

In this paper, we have demonstrated a new spectrally-encoded color imaging method that uses a single light beam. A custom grating was designed to achieve high diffraction efficiencies for the spectral bands and diffraction orders used for SEE imaging. Color SEE images of a resolution chart and excised swine mesentery were of similar quality to those obtained by a conventional color camera. By only using a single light beam, the new color SEE method can simplify the design and manufacturing of color SEE probes and systems. Remaining technical development steps towards color SEE miniature endoscopy include miniaturization of this optical setup so that it can be utilized in a driveshaft-based, rotationally scanning probe and adapting the software so that images can be visualized in real time.

\section{Funding. Canon USA}

Acknowledgment. Dukho Do is currently with Samsung Electronics (South Korea).

\section{References}

1. C. M. Lee, C. J. Engelbrecht, T. D. Soper, F. Helmchen, and E. J. Seibel, J. Biophotonics 3, 385 (2010).

2. G. Matz, B. Messerschmidt, W. Göbel, S. Filser, C. S. Betz, M. Kirsch, O. Uckermann, M. Kunze, S. Flämig, and A. Ehrhardt, Biomed. Opt. Express 8, 3329 (2017).

3. A. Porat, E. R. Andresen, H. Rigneault, D. Oron, S. Gigan, and O. Katz, Optics express 24, 16835 (2016).

4. G. W. Cheon, J. Cha, and J. U. Kang, Optics letters 39, 4368 (2014).

5. F. Stewart, B. Cox, J. Vorstius, A. Verbeni, Y. Qiu, and S. Cochran, 2015 IEEE International, (IEEE, 2015), 1.

6. G. J. Tearney, M. Shishkov, and B. E. Bouma, Opt. Lett. 27, 412 (2002).

7. D. Yelin, I. Rizvi, W. M. White, J. T. Motz, T. Hasan, B. E. Bouma, and G. J. Tearney, Nature 443, 765 (2006).

8. D. Kang, D. Yelin, B. E. Bouma, and G. J. Tearney, Opt Express 17, 15239 (2009).

9. A. Zeidan and D. Yelin, Biomed. Opt. Express 7, 392 (2016).

10. M. Moharam, T. Gaylord, E. B. Grann, and D. A. Pommet, JOSA a 12, 1068 (1995).

11. D. Kang, R. V. Martinez, G. M. Whitesides, and G. J. Tearney, Lab on a chip 13, 1810 (2013). 


\section{References}

1. C. M. Lee, C. J. Engelbrecht, T. D. Soper, F. Helmchen, and E. J. Seibel, "Scanning fiber endoscopy with highly flexible, $1 \mathrm{~mm}$ catheterscopes for wide-field, full-color imaging," J. Biophotonics 3, 385-407 (2010).

2. G. Matz, B. Messerschmidt, W. Göbel, S. Filser, C. S. Betz, M. Kirsch, O. Uckermann, M. Kunze, S. Flämig, and A. Ehrhardt, "Chip-on-the-tip compact flexible endoscopic epifluorescence video-microscope for in-vivo imaging in medicine and biomedical research," Biomed. Opt. Express 8, 3329-3342 (2017).

3. A. Porat, E. R. Andresen, H. Rigneault, D. Oron, S. Gigan, and O. Katz, "Widefield lensless imaging through a fiber bundle via speckle correlations," Optics express 24, 16835-16855 (2016).

4. G. W. Cheon, J. Cha, and J. U. Kang, "Random transverse motioninduced spatial compounding for fiber bundle imaging," Optics letters 39, 4368-4371 (2014).

5. F. Stewart, B. Cox, J. Vorstius, A. Verbeni, Y. Qiu, and S. Cochran, "Capsule-based ultrasound-mediated targeted gastrointestinal drug delivery," in Ultrasonics Symposium (IUS), 2015 IEEE International, (IEEE, 2015), 1-4.

6. G. J. Tearney, M. Shishkov, and B. E. Bouma, "Spectrally encoded miniature endoscopy," Opt. Lett. 27, 412-414 (2002).

7. D. Yelin, I. Rizvi, W. M. White, J. T. Motz, T. Hasan, B. E. Bouma, and G. J. Tearney, "Three-dimensional miniature endoscopy," Nature 443, 765 (2006).

8. D. Kang, D. Yelin, B. E. Bouma, and G. J. Tearney, "Spectrally-encoded color imaging," Opt Express 17, 15239-15247 (2009)

9. A. Zeidan and D. Yelin, "Spectral imaging using forward-viewing spectrally encoded endoscopy," Biomed. Opt. Express 7, 392-398 (2016).

10. M. Moharam, T. Gaylord, E. B. Grann, and D. A. Pommet, "Formulation for stable and efficient implementation of the rigorous coupled-wave analysis of binary gratings," JOSA a 12, 1068-1076 (1995).

11. D. Kang, R. V. Martinez, G. M. Whitesides, and G. J. Tearney, "Miniature grating for spectrally-encoded endoscopy," Lab on a chip 13, 1810-1816 (2013). 